\title{
Nanotopography-induced changes in focal adhesions, cytoskeletal organization, and mechanical properties of human mesenchymal stem cells
}

\author{
Evelyn K.F. Yim ${ }^{\mathrm{a}, *, 1}$, Eric M. Darling ${ }^{\text {a,b,2 }}$, Karina Kulangara ${ }^{\text {a }}$, Farshid Guilak ${ }^{\text {a,b,c }}$, Kam W. Leong ${ }^{\text {a,b,c,* }}$ \\ a Department of Biomedical Engineering, Pratt School of Engineering Duke University, Durham, NC 27708, USA \\ ${ }^{\mathrm{b}}$ Department of Surgery, Duke University School of Medicine, Duke University, Durham, NC 27710, USA \\ ${ }^{\mathrm{c}}$ Department of Mechanical Engineering and Materials Science, Pratt School of Engineering Duke University, Durham, NC 27708, USA
}

\section{A R T I C L E I N F O}

\section{Article history:}

Received 28 September 2009

Accepted 14 October 2009

Available online 30 October 2009

\section{Keywords:}

Nanotopography

Mesenchymal stem cells

Focal adhesion

Cell biomechanics

Cell-substrate interactions

Integrin

\begin{abstract}
A B S T R A C T
The growth of stem cells can be modulated by physical factors such as extracellular matrix nanotopography. We hypothesize that nanotopography modulates cell behavior by changing the integrin clustering and focal adhesion (FA) assembly, leading to changes in cytoskeletal organization and cell mechanical properties. Human mesenchymal stem cells (hMSCs) cultured on $350 \mathrm{~nm}$ gratings of tissueculture polystyrene (TCPS) and polydimethylsiloxane (PDMS) showed decreased expression of integrin subunits $\alpha 2, \alpha 6, \alpha \mathrm{V}, \beta 2, \beta 3$ and $\beta 4$ compared to the unpatterned controls. On gratings, the elongated hMSCs exhibited an aligned actin cytoskeleton, while on unpatterned controls, spreading cells showed a random but denser actin cytoskeleton network. Expression of cytoskeleton and FA components was also altered by the nanotopography as reflected in the mechanical properties measured by atomic force microscopy (AFM) indentation. On the rigid TCPS, hMSCs on gratings exhibited lower instantaneous and equilibrium Young's moduli and apparent viscosity. On the softer PDMS, the effects of nanotopography were not significant. However, hMSCs cultured on PDMS showed lower cell mechanical properties than those on TCPS, regardless of topography. These suggest that both nanotopography and substrate stiffness could be important in determining mechanical properties, while nanotopography may be more dominant in determining the organization of the cytoskeleton and FAs.
\end{abstract}

(c) 2009 Elsevier Ltd. All rights reserved.

\section{Introduction}

A crucial element of tissue engineering is to create a favorable extracellular microenvironment, mainly the extracellular matrix $(\mathrm{ECM})$, to guide cell differentiation and tissue regeneration. The ECM imparts a wealth of biochemical and biomechanical cues [1], of which the latter can be presented in the form of nanotopography and matrix stiffness [2]. Recent findings show that mammalian cells do respond to nanoscale features on synthetic surfaces $[3,4]$. Our previous studies show that nanotopography can significantly influence cellular behavior ranging from morphological changes to differentiation. For example, we have demonstrated that

\footnotetext{
* Corresponding authors. Department of Biomedical Engineering, Pratt School of Engineering Duke University, Durham, NC 27708, USA.

E-mail address: kam.leong@duke.edu (K.W. Leong).

1 Present address: Division of Bioengineering, National University of Singapore, Faculty of Engineering, 7 Engineering Drive 1, Singapore 117574.

2 Present address: Department of Molecular Pharmacology, Physiology, \& Biotechnology, Division of Biology and Medicine, Brown University, Providence, RI 02912, USA.
}

nanotopography alone can upregulate the neuronal markers of human mesenchymal stem cells (hMSCs) [5]. A recent study has also demonstrated the important roles of topography in onedimensional and three-dimensional cell migration [6].

In addition to topography, the extracellular microenvironment may also provide signaling cues to the anchorage-dependent cells via a feedback of local matrix stiffness [7]. Matrix elasticity can direct hMSCs to differentiate into specific lineages: a soft matrix induces a neurogenic phenotype, while increasingly stiffer matrices induce myogenic and osteogenic phenotypes accordingly [8]. Taken together, the observations of nanotopography-induced and stiffness-directed differentiation suggest that physical interactions between the cells and the extracellular environment, either in the form of topography or stiffness, or the combination thereof, can modulate cell function and stem cell differentiation [2].

Increasing evidence indicates that cellular interaction with the ECM plays a critical role in regulating cell proliferation, differentiation, gene expression and signal transduction [9-11]. At the cellmatrix interface, the mechanical force interaction between the cell and ECM occurs through the focal adhesions (FAs), which link the ECM to the contractile cytoskeleton, thereby activating FA signaling 
pathways [10]. The focal adhesion kinase (FAK) signal, one of the FA-activated signaling pathways, can mediate mechanotransduction by responding to both substrate rigidity and cytoskeletal tension [12,13]. Meanwhile, mechanical signals from the ECM may also be transmitted via integrin receptors through the cytoskeleton to the nucleus [14].

A number of techniques have been used to study the influence of cell-matrix interactions on cell mechanical properties. Examples include growing cells on microarrays of posts to quantify the traction forces exerted [12] and on substrates of varying mechanical stiffness to compare cell behavior [15,16]. One sensitive method for measuring the cellular and cytoskeletal mechanical properties is atomic force microscopy (AFM). This technique is based on using an ultra-sharp [18] or a spherical [19] tip attached to a flexible cantilever, which is controlled in $\mathrm{x}_{-}, \mathrm{y}-$, and $\mathrm{z}$-directions by piezoactuators. This technique has been used to quantify the properties of a variety of mesenchymal-derived cells $[20,21]$ and has been used to examine relationships between cytoskeletal tension and cell stiffness [22].

The mechanisms by which nanotopography and stiffness can direct stem cell differentiation remains unanswered. We hypothesize that changes in the FA assembly in response to the extracellular topography lead to changes in cytoskeletal structure, and ultimately to changes in cellular properties and their ability to transduce mechanical signals from the ECM. Changes in cell-ECM interactions are often associated with changes in the expression of integrins and molecules in the FA plaque, which in turn can influence F-actin organization and thus the mechanical properties of the cell properties [17]. In this study, we therefore examined nanotopography-induced changes in the expression and organization of integrins, focal adhesions, and cytoskeleton of hMSCs. Using AFM, we also investigated the effects of nanotopography on the elastic and viscoelastic properties of these cells. Understanding the underlying mechanism of how these biomechanical cues influence cell behavior would be valuable for optimization of stem cell differentiation and the design of tissue engineering scaffolds.

\section{Materials and methods}

\subsection{Production of nanogratings with soft lithography and embossing}

The nanopattern was reproduced on poly(dimethylsiloxane) (PDMS) using soft lithography on a nanoimprinted poly(methyl methacrylate) (PMMA)-coated Si master mold [4]. The gratings on the nanoimprinted PMMA master molds were $350 \mathrm{~nm}$ in depth with two different lateral dimensions: one with $350 \mathrm{~nm}$ width and $700 \mathrm{~nm}$ pitch and another one with $500 \mathrm{~nm}$ width and $1 \mu \mathrm{m}$ pitch. The $350 \mathrm{~nm}$ gratings were used in the integrin expression analysis, while the $500 \mathrm{~nm}$ gratings were used in the cell mechanical analysis and the rest of the study, because of a technique developed in our lab that allowed direct embossing of the $500 \mathrm{~nm}$ gratings into commercially available $35 \mathrm{~mm}$ tissue-culture polystyrene (TCPS) dishes. Studies on TCPS have the advantage of direct comparison with the literature, with only topography as the only variable. TCPS nanopatterns were fabricated by nano-embossing pre-patterned PDMS master onto a TCPS strip or a $35 \mathrm{~mm}$ TCPS dish at a pressure of $\sim 0.5-1 \mathrm{MPa}$ and $120^{\circ} \mathrm{C}$. The PDMS samples were coated with bovine collagen I (BD Biosciences) at $20 \mu \mathrm{g} / \mathrm{cm}^{2}$ to improve cell adhesion on the hydrophobic PDMS surface. A heat-embossed unpatterned TCPS strip with collagen coating was used as a control surface when measuring the cellular mechanical properties.

\subsection{Characterization: scanning electron microscopy (SEM) and atomic force microscopy (AFM)}

The SEM samples were sputter-coated with a $5 \mathrm{~nm}$ coating of chromium and viewed with a LEO FESEM (LEO 1550) (LEO Electronic Microscopy Inc.) at $1 \mathrm{kV}$. Atomic force microscopy surface characterization was performed using contact mode scanning probe microscope (Digital Instrument Dimension 3100) in the Shared Materials Instrumentation Facility at Duke University. Static water contact angles were measured by a goniometer.

\subsection{Human mesenchymal stem cell culture}

Human mesenchymal stem cells (Poietics ${ }^{\mathrm{TM}}$ hMSCs from human bone marrow $\mathrm{CD}_{105}{ }^{+}, \mathrm{CD}_{166}{ }^{+}, \mathrm{CD} 29^{+}, \mathrm{CD} 44^{+}, \mathrm{CD} 14^{-}, \mathrm{CD} 34^{-}, \mathrm{CD} 45^{-}$, Cambrex, $\mathrm{NJ}$ ) were cultured and expanded in MSCGM medium (Cambrex). hMSCs used in the experiments were at passages 6-9. The hMSCs were seeded on all surfaces at a density of $6 \times 10^{3}$ cells $/ \mathrm{cm}^{2}$.

\subsection{Immunofluorescent staining}

Samples were fixed in $4 \%$ paraformaldehyde, permeablized with $0.1 \%$ triton $\mathrm{X} 100$ in phosphate buffered saline (PBS), and then blocked with $10 \%$ goat serum in PBS. The primary antibodies used were mouse anti-vinculin antibody diluted 1:400 (Chemicon) in tris-buffered saline (TBS) or rabbit anti-phosphorylated focal adhesion kinase (pFAK, pY397) antibody diluted 1:500 (Covance). The secondary antibodies used were Alexa-Fluor546 goat anti-mouse antibody at 1:750, or AlexaFluor488 goat anti-rabbit antibody at 1:750 (Molecular Probes). F-actin was fluorescently labeled in fixed samples with Oregon Green 488 phalloidin (Molecular Probes, Eugene, OR) as previously described [4], and the nucleus was stained with 4',6-Diamidino-2-phenylindole (DAPI, Molecular Probes). Samples were imaged by fluorescence or confocal microscopy (Zeiss LSM 410).

\subsection{Western blotting: integrin binding, focal adhesion and cytoskeleton analysis}

Protein was collected from hMSC-seeded samples using two different methods. Whole cell lysis was collected to analyze the expression of focal adhesion components and the cytoskeleton content. The integrin expression at the cell-substrate interface was analyzed by an integrin binding assay adapted from a protocol of Garcia et al. [23]. Briefly, the hMSCs were washed three times in Dulbecco's PBS and incubated in a $1 \mathrm{~mm}$ cell-impermeable sulfo-BSOCOES cross-linker (Pierce) for $15 \mathrm{~min}$ at $4{ }^{\circ} \mathrm{C}$. After quenching unreacted cross-linker with $50 \mathrm{~mm}$ Tris, cells were extracted in $0.1 \%$ SDS, $350 \mu \mathrm{g} / \mathrm{ml}$ phenylmethylsulphonyl fluoride (PMSF), $10 \mu \mathrm{g} / \mathrm{ml}$ leupeptin, and $10 \mu \mathrm{g} / \mathrm{ml}$ aprotinin. Protein cross-linked to the dish was recovered by reversing the cross-linking in $50 \mathrm{~mm} \mathrm{NaHCO}_{3}(\mathrm{pH} 11.6)$ and $0.1 \% \mathrm{SDS}$ at $37{ }^{\circ} \mathrm{C}$ for $2 \mathrm{~h}$ and concentrated by size exclusion filtration. Recovered integrins were quantified by Western blotting with $\alpha$-integrin screening kit and $\beta$-integrin screening kit (Chemicon) as primary antibodies and horseradish peroxidase-conjugated secondary antibodies (BioRad). Integrin $\beta 1$ expression was used to normalize for the differences in cell number among different samples.

For analysis of focal adhesion and cytoskeleton component content, whole cell lysis was collected. Cells were washed in TBS and lysed in cold modified RIPA buffer (50 mm Tris- $\mathrm{HCl}$, pH 7.4, 1\% Igepal CA-630, 0.25\% deoxycholate, $150 \mathrm{~mm} \mathrm{NaCl}, 1 \mathrm{~mm}$ EDTA, 1 mm PMSF, $1 \mathrm{~mm}$ orthovanadate, $1 \mathrm{~mm} \mathrm{NaF}$, and $1 \mu \mathrm{g} / \mathrm{ml}$ each aprotinin, leupeptin, and pepstatin). Proteins were separated by denaturing SDS-PAGE electroblotted onto PVDF, blocked with $5 \%$ milk and $10 \%$ goat serum in TBS, immunoblotted with specific primary antibodies: mouse anti-vinculin antibody diluted 1:400 (Chemicon), mouse anti-FAK, non-phosphorylated, diluted 1:100 (MBL International), rabbit anti-pFAK antibody diluted 1:500 (Covance), mouse anti- $\alpha$ tubulin diluted 1:50 (DSHB), mouse anti- $\beta$-actin diluted 1:5000 (Sigma), rabbit anti-actin diluted 1:200 (Sigma) and mouse anti-GAPDH diluted 1:800 (Chemicon), and detected using horseradish peroxidase-conjugated secondary antibodies (BioRad) and ECL Plus (GE Healthcare) as a chemiluminescent substrate. GAPDH was used as a loading control. Densitometric analysis was performed using an Alpha-Innotech imaging system (Fluorchem FC2).

\subsection{Atomic force microscopy analysis of mechanical properties}

The mechanical properties of single cells were measured using an atomic force microscope (MFP-3D, Asylum Research, Santa Barbara, CA) via elastic and viscoelastic tests as described previously [21]. Borosilicate glass spheres (5 $\mu \mathrm{m}$ diameter) were attached to the tip of AFM cantilevers $(\mathrm{k} \sim 0.04 \mathrm{~N} / \mathrm{m}$, Novascan Technologies, Inc., Ames, IA) and used for indentation and stress relaxation experiments. Indentation was performed over the cell nucleus at $15 \mu \mathrm{m} / \mathrm{s}$, a rate chosen to approximate a step displacement appropriate for the stress relaxation model. Elastic curves were sampled at $2 \mathrm{kHz}$, while viscoelastic curves were collected at $200 \mathrm{~Hz}$ for 60 seconds A 2-3 $\mathrm{nN}$ force trigger was used to prescribe the point at which the cantilever approach was stopped and either retracted for indentation tests or held constant for stress relaxation tests.

The elastic modulus, $E_{\text {elastic, }}$ was extracted from force vs. indentation data using a thin-layer Hertz model that accounts for cell thickness [24]. Probe-cell contact was identified using contact point extrapolation, a method that focuses on the indentation portion of the approach curve to determine where indentation begins [19]. The parameters $E_{R}, E_{0}$, and $\mu$, were determined using a thin-layer, stress relaxation model of a viscoelastic solid [20]. The Poisson's ratio $(\nu)$ for all cells was assumed to be 0.5 , and parametric studies showed that varying $\nu$ from 0.3 to 0.5 altered the measured properties by less than $20 \%$. 


\subsection{Statistical analysis}

Data on cell mechanical properties ( $n>32$ per group) were not normally distributed according to the Shapiro-Wilks test, and were therefore log-transformed before statistical analysis. Single-factor ANOVA with Fisher LSD post-hoc analysis was performed using the Statistica software package (StatSoft, Tulsa, OK) to determine whether significant differences $(\alpha=0.05)$ in cellular mechanical properties existed among substrates. Mechanical properties are reported as mean \pm standard error.

\section{Results}

\subsection{Nanopatterned TCPS and PDMS}

The difference in the material properties of PDMS and TCPS allowed us to investigate the effects of different materials along with topography. The Young's modulus was around 2-4 MPa for PDMS [25] and 2 GPa for PS [26]. Their corresponding static contact angles without collagen coating were $95-100^{\circ}$ and $56-66^{\circ}$, respectively. AFM and SEM confirmed the quality and fidelity of the PDMS and TCPS samples, with feature size and sharp edges preserved throughout the replication process (Fig. 1). The PDMS embossing master could be re-used $>50$ times without comprising the quality of the embossed patterns.

\subsection{Integrin expression}

The densitometry data of the integrin expression were normalized with $\beta 1$ expression. The normalized expression of integrin $\alpha 1$, $\alpha 2, \alpha 4, \alpha \mathrm{V}, \beta 2, \beta 3$ and $\beta 4$ of hMSCs cultured on nanopatterned substrates was reduced compared to unpatterned controls, despite the disparity of stiffness among TCPS and PDMS (Fig. 2). In addition to the reduced expression on nanotopography, integrin expression of hMSCs cultured on the softer PDMS was also lower than on TCPS. Integrin $\alpha 4$ and $\alpha 5$ expression was not detected.

A reverse trend, however, was observed on the integrin $\alpha 3$ expression, where the $\alpha 3$ expression was higher on the nanogratings compared to unpatterned controls on either PDMS or TCPS. Integrin $\beta 5$ also exhibited a different expression pattern, which could be reflecting a combined effect of topography and stiffness.
Taken together, the data suggest that both nanotopography and substrate stiffness have altered the substrate-bound integrin expression at the cell-substrate interface.

\subsection{Cytoskeleton and focal adhesion rearrangement}

A dense network of F-actin was observed on the hMSCs cultured on the unpatterned surfaces, while parallel stress-fiber with lower density was observed on the elongated cells on the nanogratings (Fig. 3A-B). As similar cytoskeletal structure was observed on both TCPS and PDMS, it suggests that nanotopography exerts a more significant influence than substrate stiffness on the organization of the F-actin cytoskeleton.

The focal adhesions and their distribution as visualized by immunofluorescence staining of phospho-Y397-FAK (pFAK) and vinculin could be observed clearly for cells cultured on unpatterned controls (Fig. 3B and C, respectively). The pFAK could be found both at the central region and the peripheral region, at the end of the Factin fiber bundles in the filopodia or lamellipodia. In contrast, a higher density of FAs was observed at the poles of the elongated hMSCs cultured on the nanogratings.

Vinculin is a protein recruited from the cytoplasm to the focal adhesion complex. Both cytoplasmic vinculin and focal adhesion complex-recruited vinculin were stained. The cytoplasmic vinculin was observed throughout cells cultured on patterned or unpatterned surfaces. The distribution of the focal adhesion-recruited vinculin was similar to that observed in FAK staining. Again, the data showed that nanotopography elicited a more obvious difference in focal adhesion distribution than substrate stiffness when compared to the respective unpatterned controls.

\subsection{F-actin and focal adhesion expression}

The total FAK level expressed by hMSCs was lowered on both TCPS and PDMS nanopatterns compared to unpatterned controls (Fig. 4). However, the level of Y397-FAK phosphorylation was also lowered on unpatterned TCPS and PDMS. Regarding vinculin expression, on TCPS a higher level could be detected on the nanopatterned samples

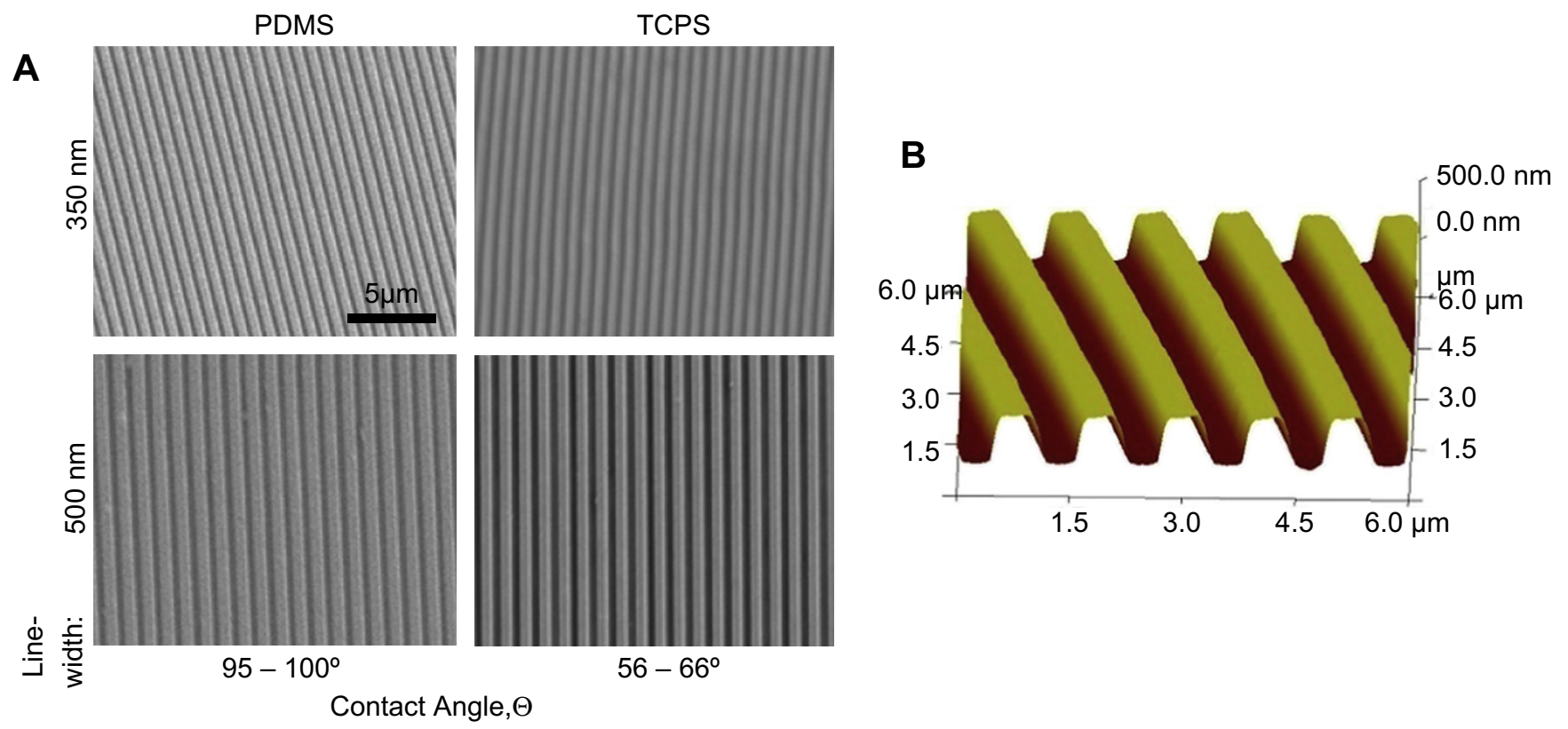

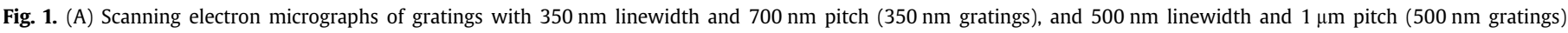

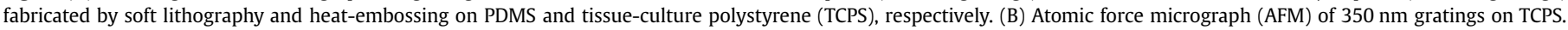




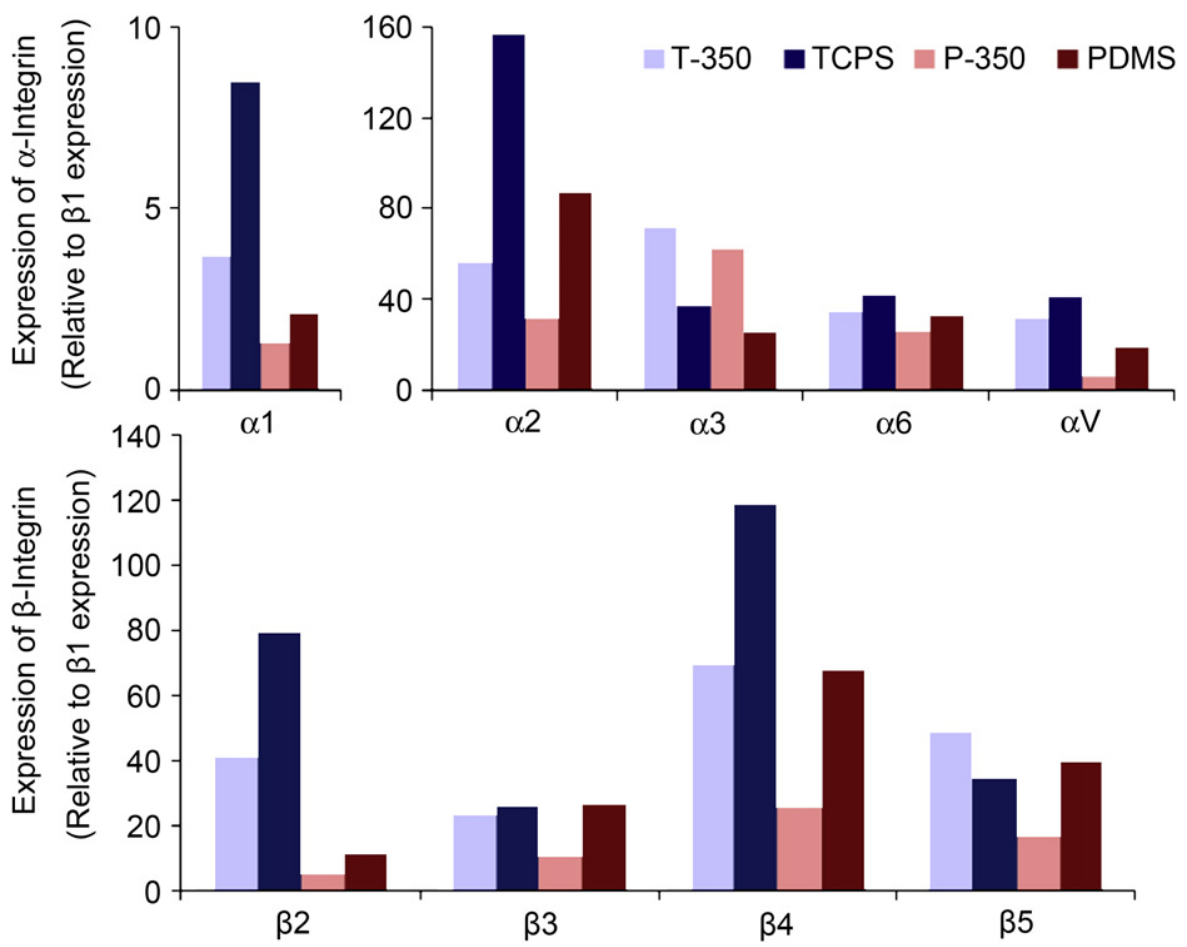

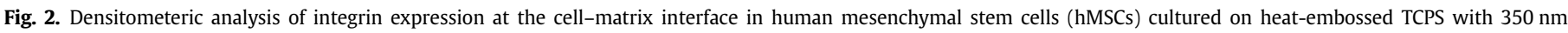
gratings (T-350) or unpatterned control (TCPS), or PDMS with $350 \mathrm{~nm}$ gratings (P-350) or unpatterned control (PDMS).

compared to the unpatterned controls. On PDMS, however, the difference was not noticeable. When the expression of cytoskeleton components was examined, the level of actin and smooth muscle actin was reduced in the hMSCs on the nanopatterned substrates compared to unpatterned controls, either on TCPS or PDMS. The microtubule $\alpha$-tubulin expression was higher in on both nanopatterns.

\subsection{Mechanical properties of hMSCs on nanopatterns}

The changes in cytoskeletal organization were reflected in the cellular mechanical properties (Fig. 5). On TCPS, the cells exhibited lower elastic moduli when attached to nanopatterned surfaces as compared to unpatterned controls either without collagen coating $(p<0.0003)$ or with collagen coating $(p<0.001)$ (Supplementary Fig. S1). On PDMS, no significant differences between surfaces existed $(p=0.14)$. However, elastic moduli for cells attached to nanopatterned and unpatterned PDMS were significantly lower than for cells attached to unpatterned TCPS $(p<0.0001)$.

The viscoelastic properties associated with cells attached to the nanopatterned surfaces showed changes that paralleled those observed in the elastic properties. The relaxed and instantaneous moduli were lower for cells on PDMS surfaces and nanopatterned TCPS compared to unpatterned TCPS $(p<0.002)$. The apparent viscosity was significantly higher for cells attached to unpatterned TCPS compared to unpatterned PDMS $(p<0.003)$, but not compared to patterned PDMS $(p=0.30)$ or patterned TCPS $(p=0.19)$. For the instantaneous modulus and apparent viscosity, no significant differences were found between cells attached to nanopatterned and unpatterned PDMS $(p>0.10)$. However, cells on nanopatterned TCPS were significantly softer than on the unpatterned equivalent $(p<0.002)$.

\section{Discussion}

The findings of this study show that both nanotopography as well as the mechanical properties of the substrate can have a significant effect on interactions between stem cells and their ECM, influencing focal adhesion formation, the organization of the cytoskeleton, and cellular mechanical properties. These findings are consistent with previous studies, which have further have spurred efforts to understand the underlying mechanisms by which such factors influence cell growth and differentiation. For example, Doyle et al. demonstrate the role played by topography in the migration of fibroblasts on a one-dimensional fibrillar pattern and three-dimensional matrix [6]. Tzvtkova-Chevolleau et al. study the migration of 3T3 fibroblasts and SaI/N fibrosacromas on micropatterns with stiffness ranging from 500-2000 kPa. Morphology and spreading are influenced by topography while the migration of SaI/N fibrosacromas is correlated with substrate rigidity [24]. Cavalcanti-Adam et al. observe that the spacing between integrin clusters regulates cell spreading in fibroblasts [27]. In the current study, we also find that the nanotopography of the substrate influenced integrin expression in hMSCs. The expression of integrin $\alpha 1, \alpha 2, \alpha 6, \alpha \mathrm{V}, \beta 2, \beta 3$ and $\beta 4$ is reduced in hMSCs cultured on nanogratings compared to either unpatterned PDMS or TCPS. The consistent trend of integrin downregulation on these two substrates with vastly different mechanical properties suggests that this is predominantly a nanotopography-mediated phenomenon.

Integrin-mediated adhesion to the ECM plays an important role in regulating cell behavior [28]. The transmembrane integrin binds to the extracellular substrate on one side, and binds to linker proteins of focal adhesion such as vinculin that connect the integrin molecules to actin stress-fibers within the cell. The integrin-ECM binding facilitates cell contact and initiates the assembly of focal adhesions while activating the FAK and focal adhesion-associated kinase simultaneously. FAK in turn also mediates other pathways such as RhoA, which stimulates the actomyosin contraction [29] and proliferation, [30], and Rac1, which regulates lamellipodia formation [31]. Given the important roles of ECM-integrin binding, our observation of the nanotopography-mediated changes in integrin expression suggests that nanotopographical modulation of 

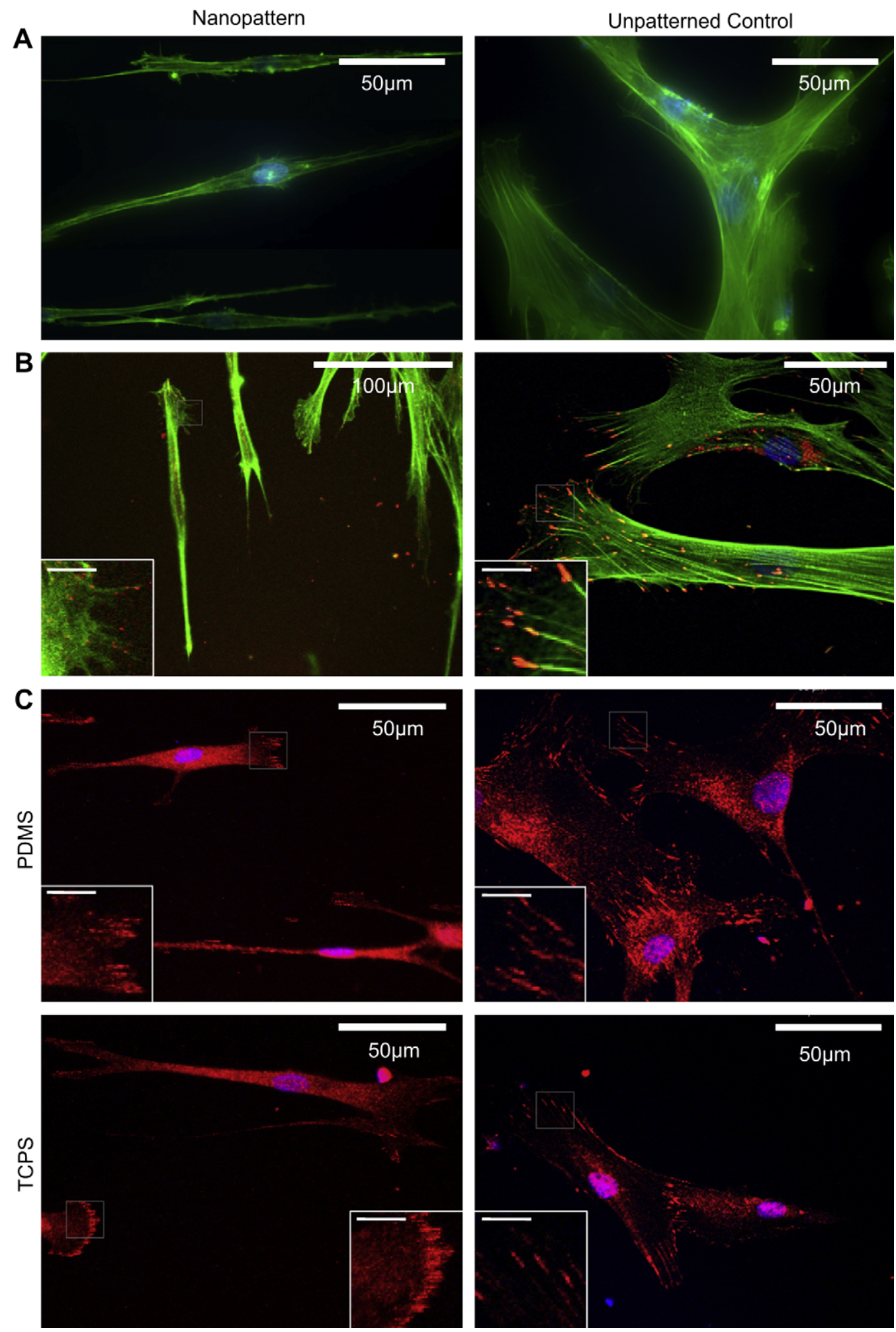

Fig. 3. (A) F-actin cytoskeleton visualized by Oregon green labeled phallodin in hMSC on PDMS with $350 \mathrm{~nm}$ gratings or unpatterned PDMS. (B) Distribution of focal adhesions visualized by immunofluorescence staining of tyrosine-397 phosphorylated FAK (pFAK, red) and F-actin (green). (C) Distribution of focal adhesions visualized by immunofluorescence staining of vinculin (red). Grey boxes indicate the area of the magnified views shown in the insert figures; $b a r=10 \mu \mathrm{m}$ in the insert figures. Images of (A) are taken with fluorescence microscopy; images of $(\mathrm{B}-\mathrm{C})$ are taken with confocal microscopy.

cell behavior could be initiated with the regulation of integrin clustering and, subsequently, the FA assembly and FAK activation.

Furthermore, the integrins and the FAs may also serve as mechanosensors that transmit and translate applied or internal actin forces in the form of cell signaling [32-34]. The dynamics of the growth of focal adhesion is driven by cytoskeletal forces and the availability of additional cytoplasmic proteins. Forces that activate the stabilization and growth of focal adhesion can be generated 
A

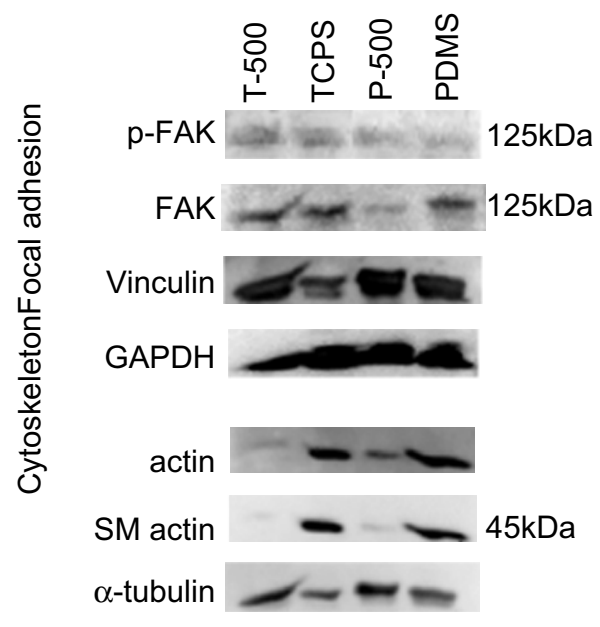

B

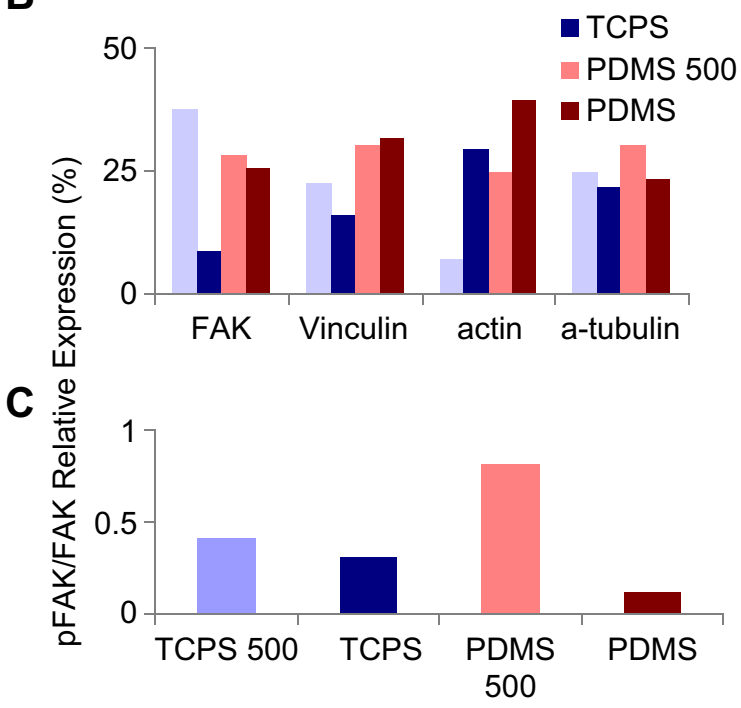

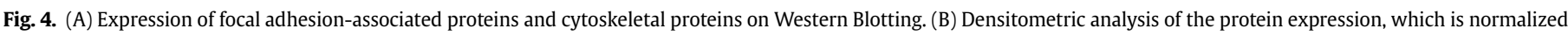
to the GAPDH loading controls. (C) Phosphorylation level of FAK in hMSCs cultured on different surfaces.

internally by the intracellular contractile actin-myosin-II machinery, or applied externally [10]. The characterization of FA, namely FAK and vinculin, and cytoskeleton such as actin arrangement would provide evidences on how topography affects the mechanotransduction of the cell. This study focuses on the steady state regulation of topography on FA and cytoskeleton arrangement. We observe that the FAK and vinculin expressions in the elongated hMSCs differ from those on unpatterned controls. The focal adhesions, as visualized by focal adhesion-recruited vinculin and $\mathrm{pFAK}$, are distributed over the peripheral and central regions of the spread hMSCs on unpatterned surfaces but localized at the poles of the elongated hMSCs on the nanogratings. Accompanying this distribution pattern is a significant reduction in cytoskeleton protein expression, the expression of the force-bearing actin, in the hMSCs on the nanopatterned substrates. Mechanical force can activate connections between cytoskeleton and focal adhesion signaling, enabling the cell to sense and respond to the extracellular stimuli. It is easier for the cells to develop a higher cytoskeletal tension on a stiffer substrate, where the cytoskeletal force can be counter-balanced; hence the transmission of forces from the ECM to the cell may be influenced by substrate rigidity [35,36]. Taken together, these findings suggest that the changes in the morphology of FA and cytoskeleton arrangement in this study may be related to the cellular force sensing of ECM and the cytoskeletonFA feedback regulation of the externally applied forces due to topography and stiffness.

Cell-shape regulation of proliferation, which is an essential mechanism in embryonic development and wound healing, could be another mechanism in mediating cell behavior. In recent studies, micropatterned adhesive islands have been used to independently control cell spreading and elongation [37]. Similar to our findings, cell elongation induced by the fibronectin-coated micropattern affects the organization of actin cytoskeleton, reducing both cytoskeletal stiffness and contractility. Despite the reduction in cytoskeletal stiffness, a moderate increase of F-actin content is observed in the elongated cells with aligned actin filament in their study. Because the cytoskeletal stiffness and contractility is often related to either F-actin content, formation of stress-fibers and/or the spatial organization of the actin cytoskeleton, the authors propose that the reduced stiffness observed in the elongated cells is related to the altered structure of the cytoskeleton. The actin microfilament cross-linking would be reduced in a parallel stress-fiber network in elongated cells, compared to the denser cross-linking in an entangled stress-fiber network in spread endothelial cells. Thus, the authors suggest that the spatial organization of the actin cytoskeleton plays an important role in maintaining the cell mechanics [37]. Consistent with these previous findings on the endothelial cells, our AFM indention study also shows a reduced stiffness in the elongated hMSCs, suggesting that the spatial organization of actin filaments can be a determining factor for cell stiffness.

In another study, Saez et al. vary the substrate stiffness anisotropically by using micro-pillars of different geometry [38]. They find that anisotropic stiffness on elliptical pillars induces the elongation and directional migration of Madin-Darby canine kidney (MDCK) epithelial cells. The elongation and directional movement is observed on PDMS pillars but not on PS pillars. The

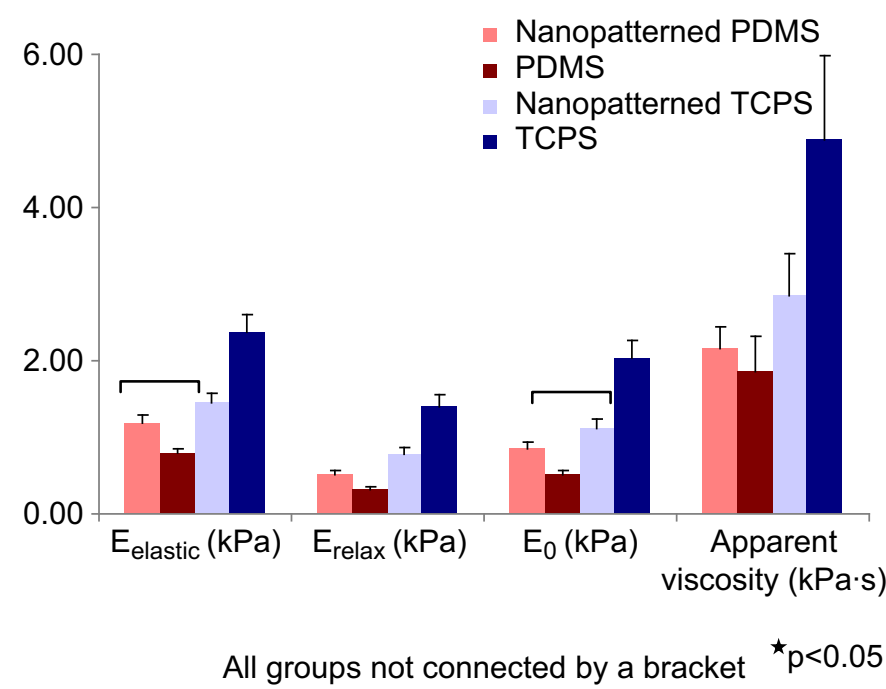

Fig. 5. Mechanical properties of hMSCs cultured on different surfaces as measured by AFM indentation. Results indicate a dramatic effect of the surfaces on the elastic modulus $\left(E_{\text {elastic }}\right)$, relaxed modulus $\left(E_{\text {relax }}\right)$, instantaneous modulus $\left(E_{0}\right)$ and apparent viscosity of attached cells. ( $p<0.05$ for all the groups not connected by the brackets). 
authors speculate that the cells cannot distinguish between the two directions of the ellipse on the PS pillars because they are too rigid in both directions, and they further suggest that the cell assembly is mainly a consequence of anisotropic substrate elasticity but not contact guidance.

Similarly, gratings can also provide an anisotropic elasticity for cells, where cells will need to exert a stronger contractile force in the direction parallel to the grating axis compared to the direction perpendicular to the grating axis. Compared to the elliptical pillars, gratings may be able to create a larger gradient of anisotropic elasticity, and/or they may be able to provide continuous contact guidance cues for the cells. Our data suggest that both topography and substrate stiffness could induce changes in cellular mechanics, in particular cytoskeleton content and rearrangement; nevertheless, only topography could induce cell anisotropy/elongation and spatial reorganization of the cytoskeletal structure, in spite of the vast difference in substrate stiffness. The discrepancy could be due to difference in geometry of the topography and the degree of anisotropic elasticity. While cell elongation, hence the change of cell-shape, may be responsible for a reduction in cytoskeleton cross-linking as suggested by Roca-Cusachs et al. [37], our data demonstrate that the additional contribution of the anisotropic elasticity created by the gratings, regardless of soft PDMS or rigid polystyrene materials, could induce the reduction in the actin content in response to a change in the external mechanical force.

\subsection{Mechanical properties}

Recent studies suggest that cellular mechanical properties may serve as novel biological markers of cell phenotypes, reflecting changes in differentiation or cellular transformation $[8,19]$. Our previous study shows that the mechanical properties of single cells are strongly associated with their lineage [19]. For example, osteoblasts exhibit stiffer moduli than chondrocytes, which in turn are stiffer than adipocytes. A recent study by Elkin et al. also suggests that neurons exhibit elastic moduli even lower than that of adipocytes [39].

We have previously shown that nanotopography could induce hMSCs to differentiate into neuronal-like phenotype, with significant increases in the expression of neuronal markers. The extent of differentiation is greater than that due to biochemical cues alone [5]. Meanwhile, previous studies have shown that substrate stiffness, in addition to biochemical stimuli, plays a role in the differentiation of hMSCs towards select lineages: osteoblastic differentiation occurring on the stiffest material, myogenic on intermediate stiffness, and neurogenic on the softest [8]. The reduced elastic and viscoelastic properties of hMSCs observed in the current study are consistent with the fact that hMSCs on nanogratings undergo neuronal differentiation and exhibit a lower elastic modulus than the other cell types.

An interesting but puzzling finding of this study is that the changes in mechanical properties of the hMSCs induced by nanotopography differ depending on the stiffness of the substrate. While nanopatterned TCPS decreases the elastic, relaxed, and instantaneous Young's moduli of cultured hMSCs, the opposite trend is observed for cells on patterned vs. unpatterned PDMS (Fig. 5). The actin cytoskeleton arrangement and FA distribution are the same for elongated cells on either rigid TCPS or soft PDMS. Cell spreading, actin cytoskeleton and FA distribution for the hMSCs attached to unpatterned TCPS and PDMS are also similar. Even though the total F-actin content is decreased and the alignment of the actin cytoskeleton is induced by nanotopography in a similar manner on the TCPS and PDMS, the anisotropic gradient of rigidity created by the nanogratings could be different on the two materials. This difference in anisotropic rigidity could in turn alter integrin clustering,
F-actin organization, and the phosphorylation levels of FAK, thereby affecting focal adhesion signaling, which would contribute to the different trends of cellular mechanics observed by AFM.

Since the decreased stiffness of hMSCs on nanopatterned TCPS could be related to the heat-embossing process; therefore, we also studied the hMSC stiffness on heat-embossed unpatterned TCPS $(n=13)$. However, the resulting cellular mechanical properties are similar to those observed for hMSCs on unprocessed TCPS (Supplement Fig. S1). Thus, the decreased stiffness of hMSCs observed on the nanopatterned TCPS is unlikely caused by the heatembossing process.

\section{Conclusions}

Our findings show that substrate-bound integrin expression, focal adhesion assembly, and F-actin content are not only affected by substrate stiffness but also by topography, and that these changes are also reflected in cell viscoelastic properties. Thus, cells sense and respond to topography and substrate stiffness, possibly via mechanotransduction through an integrin-focal adhesioncytoskeleton pathway. This transduction also appears to affect the differentiation and thus cell fate of hMSCs, suggesting that defined control of ECM nanotopography and stiffness may provide an important tool in the development of "cell-instructive" tissue engineering scaffolds that can influence stem cell behavior in a predefined manner.

\section{Acknowledgements}

This work is partially supported by NIH HL83008 for EKFY and KWL, AR53448 for EMD and AG15768, AR50245, AR48852, AR48182 for FG. The authors thank Professor S. Zauscher for the scientific discussion and for providing assistance with the atomic force microscopy measurement of cellular mechanical properties. KK would like to acknowledge Swiss National Science Foundation grant PBEL2-115950 for her financial support.

\section{Appendix}

Figures with essential color discrimination. All figures of this article are difficult to interpret in black and white. The full color images can be found in the on-line version, at doi:10.1016/j. biomaterials.2009.10.037.

\section{Appendix. Supplementary data}

Supplementary data associated with this article can be found in the online version, at doi:10.1016/j.biomaterials.2009.10.037.

\section{References}

[1] Abrams GA, Goodman SL, Nealey PF, Franco M, Murphy CJ. Nanoscale topography of the basement membrane underlying the corneal epithelium of the rhesus macaque. Cell Tissue Res 2000;299(1):39-46.

[2] Guilak F, Cohen DM, Estes BT, Gimble JM, Liedtke W, Chen CS. Control of stem cell fate by physical interactions with the extracellular matrix. Cell Stem Cell 2009;5(1):17-26.

[3] Dalby MJ, Gadegaard N, Riehle MO, Wilkinson CDW, Curtis ASG. Investigating filopodia sensing using arrays of defined nano-pits down to $35 \mathrm{~nm}$ diameter in size. Int J Biochem Cell Biol 2004;36(10):2005-15.

[4] Yim EK, Reano RM, Pang SW, Yee AF, Chen CS, Leong KW. Nanopatterninduced changes in morphology and motility of smooth muscle cells. Biomaterials 2005;26(26):5405-13.

[5] Yim EK, Pang SW, Leong KW. Synthetic nanostructures inducing differentiation of human mesenchymal stem cells into neuronal lineage. Exp Cell Res 2007;313(9):1820-9.

[6] Doyle AD, Wang FW, Matsumoto K, Yamada KM. One-dimensional topography underlies three-dimensional fibrillar cell migration. J Cell Biol 2009;184(4): 481-90. 
[7] Discher DE, Janmey P, Wang YL. Tissue cells feel and respond to the stiffness of their substrate. Science 2005;310(5751):1139-43.

[8] Engler AJ, Sen S, Sweeney HL, Discher DE. Matrix elasticity directs stem cell lineage specification. Cell 2006;126(4):677-89.

[9] Tilghman RW, Parsons JT. Focal adhesion kinase as a regulator of cell tension in the progression of cancer. Semin Cancer Biol 2008;18(1):45-52.

[10] Geiger B, Spatz JP, Bershadsky AD. Environmental sensing through focal adhesions. Nat Rev Mol Cell Biol 2009;10(1):21-33.

[11] Vogel V, Sheetz M. Local force and geometry sensing regulate cell functions. Nat Rev Mol Cell Biol 2006;7(4):265-75.

[12] Chen CS, Tan J, Tien J. Mechanotransduction at cell-matrix and cell-cell contacts. Annu Rev Biomed Eng 2004;6:275-302.

[13] Burridge K, Fath K, Kelly T, Nuckolls G, Turner C. Focal adhesions: transmembrane junctions between the extracellular matrix and the cytoskeleton. Annu Rev Cell Biol 1988;4:487-525.

[14] Itano N, Okamoto S, Zhang D, Lipton SA, Ruoslahti E. Cell spreading controls endoplasmic and nuclear calcium: a physical gene regulation pathway from the cell surface to the nucleus. Proc Natl Acad Sci U S A 2003;100(9):5181-6.

[15] Frey MT, Wang YL. A photo-modulatable material for probing cellular responses to substrate rigidity. Soft Matter 2009;5:1918-24.

[16] Yeung T, Georges PC, Flanagan LA, Marg B, Ortiz M, Funaki M, et al. Effects of substrate stiffness on cell morphology, cytoskeletal structure, and adhesion. Cell Motil Cytoskeleton 2005;60(1):24-34.

[17] Docheva D, Padula D, Popov C, Mutschler W, Clausen-Schaumann H Schieker M. Researching into the cellular shape, volume and elasticity of mesenchymal stem cells, osteoblasts and osteosarcoma cells by atomic force microscopy. J Cell Mol Med 2008;12(2):537-52.

[18] Darling EM, Topel M, Zauscher S, Vail TP, Guilak F. Viscoelastic properties of human mesenchymally-derived stem cells and primary osteoblasts, chondrocytes, and adipocytes. J Biomech 2008;41(2):454-64.

[19] Darling EM, Zauscher S, Block JA, Guilak F. A thin-layer model for viscoelastic, stress-relaxation testing of cells using atomic force microscopy: do cell properties reflect metastatic potential? Biophys J 2007;92(5):1784-91.

[20] Darling EM, Zauscher S, Guilak F. Viscoelastic properties of zonal articular chondrocytes measured by atomic force microscopy. Osteoarthr Cartil 2006;14(6):571-9.

[21] Mizutani T, Haga H, Kawabata K. Cellular stiffness response to external deformation: tensional homeostasis in a single fibroblast. Cell Motil Cytoskeleton 2004;59(4):242-8.

[22] Wang N, Ingber DE. Probing transmembrane mechanical coupling and cytomechanics using magnetic twisting cytometry. Biochem Cell Biol 1995;73(7-8):327-35.

[23] Garcia AJ, Vega MD, Boettiger D. Modulation of cell proliferation and differentiation through substrate-dependent changes in fibronectin conformation. Mol Biol Cell 1999;10(3):785-98.
[24] Dimitriadis EK, Horkay F, Maresca J, Kachar B, Chadwick RS. Determination of elastic moduli of thin layers of soft material using the atomic force microscope. Biophys J 2002;82(5):2798-810.

[25] Fuard D, Tzvetkova-Chevolleau T, Decossas S, Tracqui P, Schiavone P. Optimization of poly-di-methyl-siloxane (PDMS) substrates for studying cellula adhesion and motility. Microelectron Eng 2008;85(5-6):1289-93.

[26] Tzvetkova-Chevolleau T, Stephanou A, Fuard D, Ohayon J, Schiavone P Tracqui $P$. The motility of normal and cancer cells in response to the combined influence of the substrate rigidity and anisotropic microstructure. Biomaterials 2008;29(10):1541-51.

[27] Cavalcanti-Adam EA, Volberg T, Micoulet A, Kessler H, Geiger B, Spatz JP. Cell spreading and focal adhesion dynamics are regulated by spacing of integrin ligands. Biophys J 2007;92(8):2964-74.

[28] Giancotti FG. A structural view of integrin activation and signaling. Dev Cell 2003;4(2):149-51.

[29] Watanabe N, Kato T, Fujita A, Ishizaki T, Narumiya S. Cooperation between mDia1 and ROCK in Rho-induced actin reorganization. Nat Cell Biol 1999;1(3):136-43.

[30] Pirone DM, Liu WF, Ruiz SA, Gao L, Raghavan S, Lemmon CA, et al. An inhibitory role for FAK in regulating proliferation: a link between limited adhesion and RhoA-ROCK signaling. J Cell Biol 2006;174(2):277-88.

[31] Burridge K, Wennerberg K. Rho and Rac take center stage. Cell 2004;116(2):167-79.

[32] Besser A, Safran SA. Force-induced adsorption and anisotropic growth of focal adhesions. Biophys J 2006;90(10):3469-84.

[33] Delanoe-Ayari H, Al Kurdi R, Vallade M, Gulino-Debrac D, Riveline D. Membrane and acto-myosin tension promote clustering of adhesion proteins. Proc Natl Acad Sci U S A 2004;101(8):2229-34.

[34] Schwarz US, Balaban NQ, Riveline D, Bershadsky A, Geiger B, Safran SA. Calculation of forces at focal adhesions from elastic substrate data: the effect of localized force and the need for regularization. Biophys J 2002;83(3):1380-94.

[35] Bischofs IB, Schwarz US. Cell organization in soft media due to active mechanosensing. Proc Natl Acad Sci U S A 2003;100(16):9274-9.

[36] Pelham Jr RJ, Wang Y. Cell locomotion and focal adhesions are regulated by substrate flexibility. Proc Natl Acad Sci U S A 1997;94(25):13661-5.

[37] Roca-Cusachs P, Alcaraz J, Sunyer R, Samitier J, Farre R, Navajas D. Micropatterning of single endothelial cell shape reveals a tight coupling between nuclear volume in G1 and proliferation. Biophys J 2008;94(12):4984-95.

[38] Saez A, Ghibaudo M, Buguin A, Silberzan P, Ladoux B. Rigidity-driven growth and migration of epithelial cells on microstructured anisotropic substrates. Proc Natl Acad Sci U S A 2007;104(20):8281-6.

[39] Elkin BS, Azeloglu EU, Costa KD, Morrison 3rd B. Mechanical heterogeneity of the rat hippocampus measured by atomic force microscope indentation. J Neurotrauma 2007;24(5):812-22. 